\title{
Glassforming Liquids, Amorphous and Semicrystalline Polymers: Exploring their Energy Landscape and Dynamical Heterogeneity by Multi-frequency High-Field EPR
}

\author{
Vasile Bercu ${ }^{1}$ - Carlo Andrea Massa² - Silvia Pizzanelli ${ }^{2}$ Luca Pardi² . \\ Dino Leporini ${ }^{2,3}$. Massimo Martinelli ${ }^{4}$
}

Received: 12 June 2020 / Revised: 27 July 2020 / Published online: 28 October 2020

(c) The Author(s) 2020

\begin{abstract}
We review past and recent work carried out on viscous liquids, amorphous and semicrystalline polymers by multifrequency high-field electron paramagnetic resonance (HF-EPR) facility in Pisa. The emphasis is on the enhanced ability to provide fine details of the reorientation process of the paramagnetic guest, the spin probe, revealing features driving the dynamics of the host system, including the energy-barrier distribution of glassy polymers, the dynamical heterogeneity of semicrystalline polymers, and the dynamical changes occurring at the critical temperature predicted by the ideal mode-coupling theory.
\end{abstract}

PACS 64.70.Pf $\cdot 76.30 .-\mathrm{v} \cdot 61.25 . \mathrm{Hq}$

\section{Introduction}

Many disordered systems, like glasses, liquids, polymers, and bio-systems, which are of interest in physics, materials science, biology, and chemistry, have been studied over the years by conventional X-band $(9.5 \mathrm{GHz})$ electron paramagnetic resonance (EPR) spectroscopy $[1,2]$. However, the large variety of their local environments lead to wide distributions of both static and dynamical features of the paramagnetic

Dino Leporini

dino.leporini@unipi.it

1 Faculty of Physics, University of Bucharest, Str. Atomistilor 405, Magurele, Jud. Ilfov, Bucharest 077125, Romania

2 Istituto per i Processi Chimico-Fisici-Consiglio Nazionale delle Ricerche (IPCF-CNR), via G. Moruzzi 1, 56124 Pisa, Italy

3 Dipartimento di Fisica “Enrico Fermi”, Università di Pisa, Largo B. Pontecorvo 3, 56127 Pisa, Italy

4 Livorno, Italy 
centres with subsequent poor resolution of their EPR spectra. By employing higher and higher magnetic fields, these limitations have increasingly less impact and this aspect largely motivated the development of high-field electron paramagnetic (HFEPR) spectroscopy at $\mathrm{W}$-band $(95 \mathrm{GHz})$ or higher frequencies. Comprehensive textbooks [3], reviews [4], as well as concise introductions [5] concerning HF-EPR are available. Current applications of HF-EPR include studies concerning proteins and their model systems [3, 4], lipid membranes [4, 6], polymers [7]-e.g., the complex polymer lignin [8], conjugated polymers [9]—semiconductor nanocrystals [10], fullerenes [11], high-spin systems $(S=2)$ [12], and the development of new technologies for complex fluids [13].

The present paper reviews in a concise way the experimental efforts carried out in Pisa using the HF-EPR spectroscopy to provide novel insight into a wide class of disordered systems both in the solid state, i.e., amorphous polymers [14-20], and in the liquid state, i.e., glassforming viscous liquids [21, 22], polymer melts [21] and semicrystalline polymers $[23,24]$. For completeness, in addition to the previous studies, we also mention other investigations employing the same equipment [25-31]. The latter studies are not reviewed here.

After a period of technological development started in 1999, the HF-EPR spectrometer setup in Pisa eventually reached the final form reported elsewhere [32], superseding the previous versions [33]. Ref. [32] describes the status of the HFEPR spectrometer in 2002 with its structure, performances, and limits (see also [34, 35]). In the first decade of the century, attempts to implement a Quasi Optical setup were pursued that finally were abandoned due to the lack of appropriately consistent funding.

Two main issues were addressed, namely the distribution of energy barriers which must be overcome by the spin probe during the reorientation process [14-20] and the spatial distribution of microscopic mobility [23, 24]. The former aspect is strictly related to the features of the so-called "energy landscape" of glasses, whereas the latter, dubbed "dynamical heterogeneity" $[36,37]$ is a distinctive feature of viscous liquids approaching the solidification process, known as glass transition, and is also present in semicrystalline polymers due to coexistence of liquid and solid fractions.

The next section presents the background concisely.

\section{Background}

HF-EPR provides insight into disordered systems due to: (1) the remarkable orientation resolution of the spin probe dynamics [38], to be ascribed to the larger magnitude of the anisotropic Zeeman interaction which in turn leads to a wider distribution of resonance frequencies [1, 2], (2) the use of spin probes, being well coupled to the guest phase, e.g., see [39]. 


\subsection{Glasses and Liquids}

The solidification of a viscous liquid in a disordered glass is a process-usually referred to as "glass transition" (GT) - where crystallization is largely or totally inhibited by suitable cooling, compression, or even chemical reaction [40-43]. On approaching GT from, e.g., higher temperatures, one observes in a limited range a spectacular decrease of the microscopic diffusivity of several orders of magnitude. The mobility loss is signaled by the corresponding huge increase of the viscosity. Interestingly, the familiar Stokes-Einstein (SE) relationship between diffusivity and the viscosity breaks down close to GT $[44,45]$. The SE failure is considered as one of the most prominent signatures of the so-called dynamical heterogeneity (DH), i.e., the spatial distribution of regions with different mobility $[36,37,46]$.

The development of a microscopic theory of GT based on first principles and no phenomenological assumption is still a subject of intense studies carried out on both atomic and molecular systems, including polymers [47]. A current tool providing a topographic view of viscous liquids and glass formation is the multidimensional landscape of the collective potential-energy hypersurface created when a large number of particles interact with one another. This potential-energy landscape (PEL) is formed by individual "basins", each containing a local potential-energy minimum or "inherent structure", corresponding to some particle arrangement. Transitions between inherent structures involve a series of activated jumps over energy barriers with distribution $g(E)$ [48]. Depending on the temperature, different parts of PEL are sensed. At high temperature, the system explores the upper limit of the energy landscape, and using the arguments provided by the Central Limit theorem, one can expect to find a Gaussian shape for $g(E)$. By cooling to the lowest temperatures, the system will begin to explore the deepest lower energy states which are expected to be exponentially distributed following the general arguments of extreme-value statistics [49].

If the particle rearrangements are thermally activated, there is an average (trapping) time $\tau=\tau_{0} \exp (E / k T)$ before overcoming the barrier of height $E$ at temperature $T, k$, and $\tau_{0}$ being the Boltzmann constant and a microscopic time scale, respectively. Therefore, the energy-barrier distribution $g(E)$ leads to a trapping time distribution $\rho(\tau)$ [14, 15]. If $g(E)$ is Gaussian, the distribution $\rho(\tau)$ takes the form of a log-Gauss distribution (LGD). If the distribution of barrier heights is exponential with width $\bar{E}$, namely:

$$
g(E)= \begin{cases}0 & \text { if } E<E_{\min } \\ \frac{1}{\bar{E}} \exp \left(-\frac{E-E_{\min }}{\bar{E}}\right) & \text { if } E \geq E_{\min }\end{cases}
$$

one finds that $\rho(\tau)$ is expressed by the power-law distribution (PD):

$$
\rho_{P D}(\tau)= \begin{cases}0 & \text { if } \tau<\tau_{P D} \\ x \tau_{P D}^{x} \tau^{-(x+1)} & \text { if } \tau \geq \tau_{P D}\end{cases}
$$

with $x=k T / \bar{E}$ and $\tau_{\mathrm{PD}}=\tau_{0} \exp \left(E_{\min } / k T\right)$. Note that the absence of energy barriers below $E_{\min }$ does not change the shape of $\rho_{\mathrm{PD}}(\tau)$ and allows for the temperature dependence of $\tau_{\mathrm{PD}}$. If the width of the energy-barrier distribution is vanishingly small, a single reorientation time (SRT) is found with: 


$$
\rho_{\mathrm{SRT}}(\tau)=\delta\left(\tau-\tau_{\mathrm{SRT}}\right)
$$

\subsection{Semicrystalline Polymers}

HF-EPR spectroscopy was used to investigate the reorientation of a spin probe in semicrystalline poly-(dimethylsiloxane) (PDMS) from the glassy region up to the melt. In semicrystalline polymers, the macromolecules pack together in ordered regions, the crystallites, which are separated by disordered noncrystalline regions. An intermediate interfacial region, usually referred to as rigid-amorphous fraction (RAF), is also present [50-52]. This region is a disordered constrained environment, whereas the rest of the noncrystalline region other than RAF is expected to exhibit properties like the completely amorphous bulk polymers and is usually termed as mobile amorphous fraction (MAF). Differently from MAF, RAF does not become liquid-like above the glass transition temperature $T_{g}$. The confinement of spin probes in the disordered fraction offers the possibility of selective studies of such regions in semicrystalline PDMS with EPR. This is one major advantage of this method considering that the assignment of a relaxation process to the amorphous, crystalline, or interfacial regions of a semicrystalline polymer is a delicate matter[53-60].

Since there is a mobility gradient from MAF to the more constrained RAF, the spin probes are expected to experience a heterogeneous dynamics, with apparent similarities with the case of viscous liquids, tracking MAF, and RAF environments. HF-EPR is more well suited than the usual X-band EPR to discriminate between different distributions of reorientation times.

We used HF-EPR to investigate another issue concerning semicrystalline polymers, i.e., reversible crystallization and melting. As a matter of fact, linear and flexible macromolecules exhibit local equilibria between the surfaces of the individual polymer crystallites and the surrounding amorphous regions which are established by thermodynamically reversible structure changes, usually referred to as reversible crystallization and melting $[51,52,61]$. This phenomenon has been ascribed to the attachment and detachment of segments of partially melted macromolecules which are held at or in the vicinity of the crystal growth face [51, 62]. In the case of PDMS, we searched signatures of an equilibrium melting/freezing local process involving RAF and MAF. However, no distinctive spectral features associated with RAF were observed in slowly cooled PDMS. Thus, we adopted an improved strategy to increase the amount of RAF by quench cooling the polymer and were able to detect an exchange process between a fraction of trapped spin probes and a more mobile one. 


\section{Glasses and Liquids}

\subsection{Glasses: Exponential Distribution of Rotational Energy Barriers in Glassy Polystyrene}

The focus of our interest was polystyrene (PS) having energy-barrier distribution $g(E)$ intensively investigated by different experimental techniques, e.g., mechanical relaxation [63], Raman [64], and light and neutron scattering [65]. Our experiments detected the HF-EPR signal of the spin probe TEMPO [2], and the results were reported in a first letter [14] and a subsequent extended paper [15]. Notice that TEMPO and the phenyl group of PS have similar shape. TEMPO is stiff with almost spherical shape [66]. It has an average van der Waals radius $r_{\text {TEMPO }}=3.3 \pm 0.2 \AA$ and may be sketched as an oblate ellipsoid with semiaxes $r_{\|} \approx 2.7 \AA$ and $r_{\perp} \approx 3.7 \AA$. Similar small spin probes are anticipated to undergo jump dynamics in glasses [67-69].

Figure 1 demonstrates that the HF-EPR line shape discriminates between different reorientation models. The PD model is more accurate. The consistency of the analysis is confirmed by Fig. 2 showing how close is the distribution of barrier heights sensed, according to the PD model, by TEMPO in glassy PS with the distributions revealed by other techniques.

Fig. 1 Best fit of the HF-EPR line shape at $190 \mathrm{GHz}$ of TEMPO spin probe in PS at $270 \mathrm{~K}$ using SRT (Eq. 3), LGD, and PD (Eq. 2). It is assumed that the spin probe undergoes reorientation by a jump angle $\phi=20^{\circ}$. Best-fit parameters for PD model: $\tau_{\mathrm{PD}}=0.225 \mathrm{~ns}$, $x=0.575$. See ref. [15] for further details

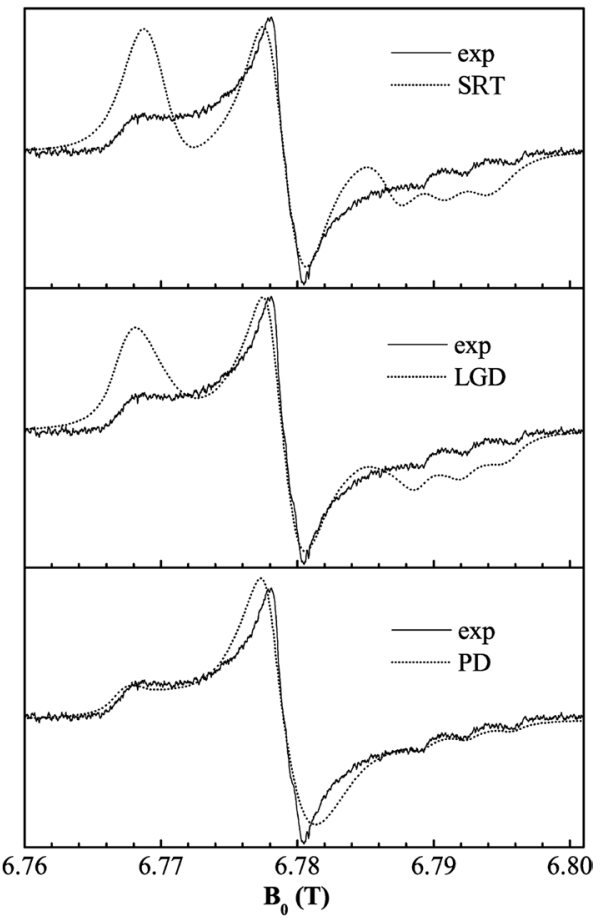




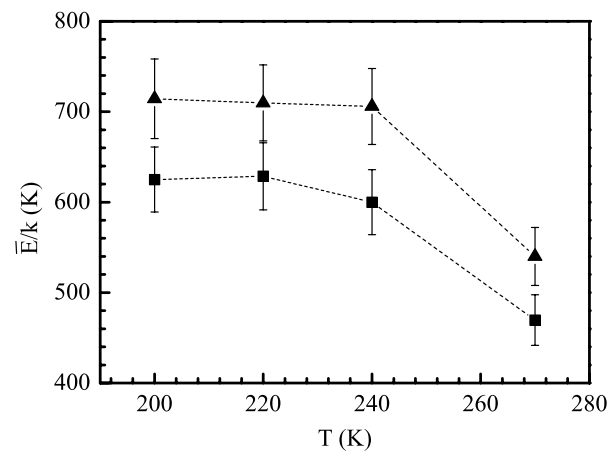

Fig. 2 Temperature dependence of the width $\bar{E}$ of the exponential energy-barrier distribution of TEMPO in glassy PS, Eq. (1), as detected by HF-EPR at $190 \mathrm{GHz}$ (squares) and $285 \mathrm{GHz}$ (triangles). Previous measurements by internal friction [63], Raman [64], and light scattering [65] yield $\bar{E}_{\mathrm{IF}} / k=760 \pm 40 \mathrm{~K}$, $\bar{E}_{\text {Raman }} / k=530 \pm 60 \mathrm{~K}$ and $\bar{E}_{L S} / k=530 \pm 40 \mathrm{~K}$, respectively. Dotted lines are guides for the eye. See ref. [15] for further details

\subsection{Liquids: Exploring the Energy Landscape Above $T_{g}$}

At temperature well above $T_{g}$, the most advanced microscopic description of the slowing down observed on approaching GT from above is the mode-coupling theory (MCT) which emphasizes the role of the cage in which the molecules are caught for a finite time due to packing constraints [70]. In particular, the ideal MCT (IMCT) predicts the existence of a sharp dynamic crossover, i.e., a transition from liquid-like to solid-like dynamics, at a critical temperature, $T_{c}$ leading to characteristic scaling laws for density correlations and, particularly, a cusp-like anomaly in the temperature dependence of the non-ergodicity parameter $f_{q}$. It has been suggested that $T_{c}$ demarcates temperatures where the system explores deeper regions of the potential-energy landscape from those at which it has access to all regions [71]. Signatures of distinct dynamical regimes in the energy landscape of a glassforming liquid have been reported [72].

Resorting to the high angular resolution of HF-EPR spectroscopy, a model independent determination of the rotational analogue $f_{\text {rot }}$ of the non-ergodicity parameter using suitable spin probes which couple to the glassy dynamics of the host was carried out [22]. The approach is based on the fact that relatively large spin probes in viscous liquids exhibit HF-EPR line shapes very close to the so-called "powder" pattern, being a distinctive feature of nearly immobile paramagnetic molecules [1, 2]. However, a closer inspection shows that even in this regime, with spin probe reorientation times roughly between 1 and $10 \mathrm{~ns}$, the line shape is highly sensitive to the specifics of the reorientation process and its rate [15]. In this respect, an elementary tool to appreciate the HF-EPR sensitivity is the distance $\Delta B$ between selected peaks of the line shape corresponding to specific extrema (or saddle points) of the resonating magnetic field of the three hyperfine components of the spectrum (turning points) $[15,20]$. 
Our system of interest was the archetypical glassformer $o$-terphenyl (OTP) where two sizeable spin probes, ANDRO and NONA, were dissolved. The study considered the temperature dependence of the spectral spacing $\Delta B_{v_{L}}$ recorded at EPR Larmor frequency $v_{L}=95,190$ and $285 \mathrm{GHz}$. Figure 3 shows the results. Four regimes are observed: regime I-at low temperatures $\Delta B_{v_{L}}$ which shows only a minor change; regime II - approaching $280 \mathrm{~K}$ a much stronger decrease of $\Delta B_{v_{L}}$ with temperature sets in which for all frequencies stops at $297.5 \pm 0.5 \mathrm{~K}$; regime III-above that temperature a plateau-like feature in $\Delta B_{v_{L}}$ vs $T$ is observed; regime IV - at the highest temperatures, again a strong decrease of $\Delta B_{v_{L}}$ is observed, reflecting the onset of the collapse of the line shape due to the faster motion of the spin probe. The collapse of the line shape is expected to occur at higher temperatures if higher Larmor frequency $v_{L}$ is employed. Consequently, the width of the plateau feature in $\Delta B_{v_{L}}$ is largest at highest frequency, and this is what is observed in regime III. In Fig. 3, the results for the probe molecule NONA are also included, showing the same qualitative behavior. Within experimental accuracy, the same crossover temperature $297.5 \pm 0.5 \mathrm{~K}$ is observed as using ANDRO spin probe, providing evidence that the phenomenon is not driven by specific features of the spin probe. The crossover temperature is rather close to the one revealed by neutron scattering at $\sim 290 \mathrm{~K}[75]$. We attribute the $\Delta B_{v_{L}}$ data for regimes I, II, and III to a pre-averaging effect due to some motion significantly faster than the structural relaxation. We interpret the decrease of $\Delta B_{v_{L}}$ as the decrease of pre-averaging effect due to the decreasing amplitude $f_{\text {rot }}$ of the fast dynamics which stops above $298 \mathrm{~K}$ and thus marks the crossover temperature $T_{c}$.

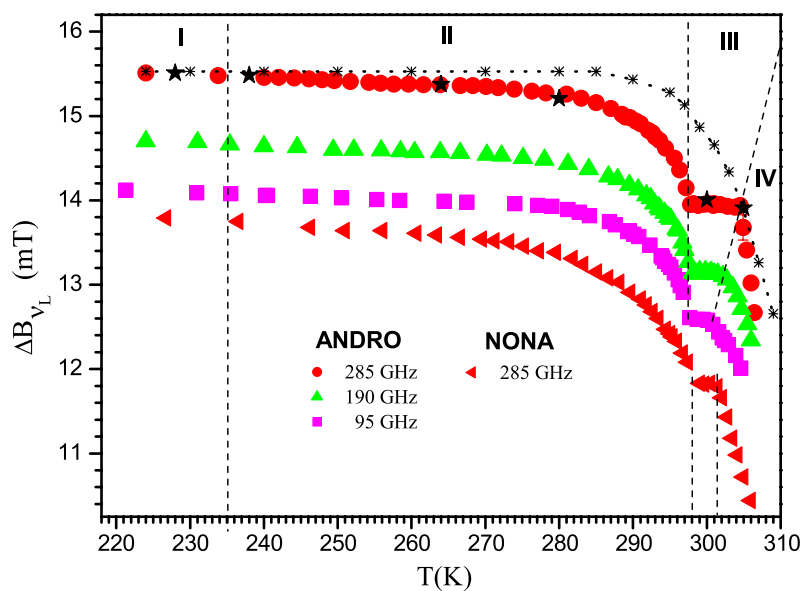

Fig. 3 Temperature dependence of $\Delta B_{v_{L}}$ of the spin probe ANDRO $\left(v_{L}=95,190,285 \mathrm{GHz}\right)$ and NONA $\left(v_{L}=285 \mathrm{GHz}\right)$ in OTP. Stars denote points concerning $\Delta B_{250}$ from ref. [73]. All curves (but the one denoted by red dots) are shifted vertically to make the comparison easier. Black curves are theoretical simulations [74] of $\Delta B_{285}$ approximating ANDRO to a prolate ellipsoid diffusing due to the $\alpha$-process with either stick (continuous) or slip (dashed) boundary conditions. Notably, neutron scattering experiments reveal the knee at $\sim 290 \mathrm{~K}$ [75], to be compared with $\sim 298 \mathrm{~K}$ revealed by HF-EPR. See ref. [22] for further details 


\section{Semicrystalline Polymers: Heterogeneous Dynamics}

\subsection{EPR Line Shapes}

The dynamics of the amorphous fraction in semicrystalline PDMS from the glassy region (below $147 \mathrm{~K}$ ) up to the melt (above about $230 \mathrm{~K}$ ) was investigated by HFEPR [23, 24]. Two different thermal protocols, slow and quench cooling, were applied to PDMS obtaining samples, PDMSsc [23] and PDMSq [24], with different amounts of RAF. In fact, slow cooling from above the melting point $\left(T_{m}\right)$ down to the temperature of interest $T\left(T_{g}<T<T_{m}\right)$ leads to less polycrystallinity than quench cooling in the glass region and subsequent reheating to reach the temperature $T$ [76-78]. The enhancement is understood in terms of both augmented primary nucleation [62] and increased disorder of the crystallite surfaces. The presence of a large number of small irregular crystallites results in a larger surface area of the crystal phase in comparison to the case of large crystallites with regular surfaces obtained upon slow cooling from the melt. The larger interface between melt and crystallites is anticipated to yield a larger amount of RAF, since the RAF thickness is weakly dependent on both the temperature and the crystallinity [50] and nanometric in size [79]. The dynamics of the amorphous fraction of the polymer was investigated monitoring the reorientation of the spin probes TEMPO and methoxy-TEMPO (m-TEMPO) in PDMSsc and PDMSq, respectively. Figure 4 shows selected HFEPR spectra of the spin probes in PDMS at different temperatures using the irradiating frequencies of 285 and $190 \mathrm{GHz}$.

The spectra markedly change with the temperature above $T_{g}=147 \mathrm{~K}$. Upon heating above $T_{g}$, the difference between the resonating magnetic field of the most distant peaks $\Delta B$ decreases and the line width of the peaks increases (see Fig. 4), until the features reminding those of the powder sample are lost around $T_{m}-20 \mathrm{~K}$ for PDMSsc. Above that temperature, the motional narrowing of the EPR line shape becomes strong, and a triplet structure starts appearing which sharpens as the temperature is increased. A similar behavior is exhibited by PDMSq, with the exception that the powder features are lost at around $T_{m}=230 \mathrm{~K}$.

\subsection{Dynamical Heterogeneity of the Tracer Reorientation}

To gain quantitative information on the spin probe reorientation, we adopted the jump model $[67,80,81]$. In PDMSsc, the spectra below $T_{g}$ were successfully simulated using the SRT model, Eq. (3), whereas above $T_{g}$, a power-law distribution of reorientation times, Eq. (2), is necessary [23]. The failure of the SRT model is anticipated in that it misses any detail on the heterogeneous dynamics occurring in the disordered region between the crystallites. Differently, in PDMSq, the SRT model satisfactorily predicts the line shape up to $200 \mathrm{~K}$ and becomes inadequate in the range $200-230 \mathrm{~K}$, where the analysis gives clear indications that the distribution of the rotational mobility of the spin probes has a bimodal structure with 
(a)

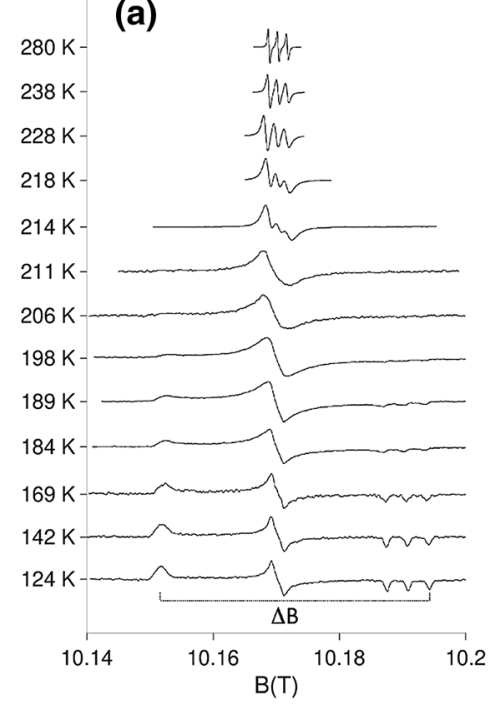

(c)

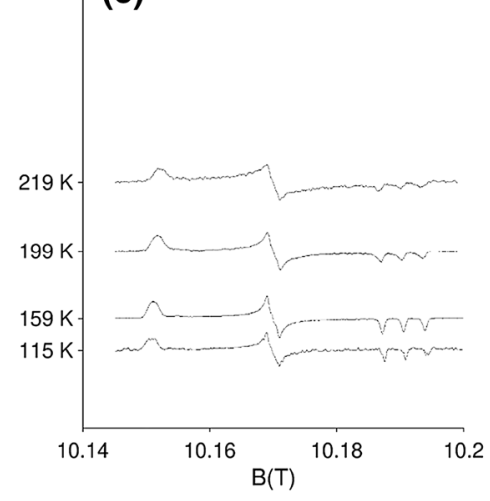

(b)

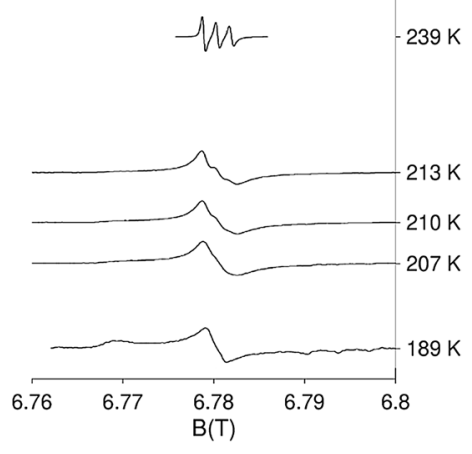

(d)

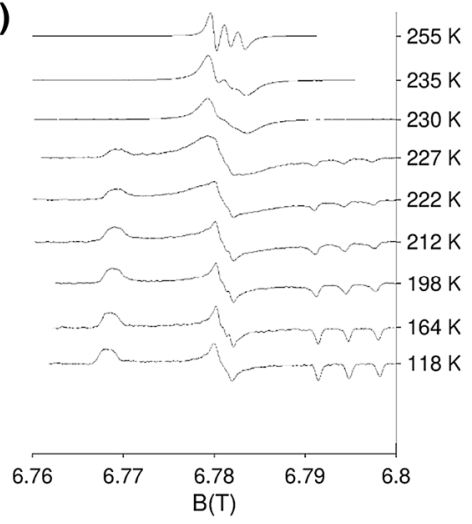

Fig. 4 Selected HF-EPR spectra of TEMPO in PDMSsc (top) [23] and of m-TEMPO in PDMSq (bottom) [24] at different temperatures using the EPR Larmor frequencies of 285 (a, c) and 190 (b, d) GHz. See $[23,24]$ for further details

(1) a broad component corresponding to spin probes with fast and intermediate mobility, as detected in PDMSsc, and (2) a narrow component corresponding to spin probes with extremely low mobility, characterized by the reorientation time $\tau_{\text {trapped }}$ [24]. The two fractions of the spin probes are expected to be located in the disordered fraction far from the crystallites and trapped close to the crystallites, respectively. In the melt, or even from $T_{m}-17 \mathrm{~K}$ for PDMSsc, as discussed below, the high PDMS fluidity averages the distribution of reorientation times quite effectively and narrows considerably the distribution, so that the description provided by the SRT model is good enough. 


\subsection{Evidence of MAF and RAF}

Figure 5 shows the temperature dependence of the average reorientation time $\langle\tau\rangle$ as drawn by the HF-EPR data sets recorded at 190 and $285 \mathrm{GHz}$ using the reorientation time distribution described above. The values of $\langle\tau\rangle$ depend little on the frequency, signaling that the whole distribution of reorientation times is collected by both frequencies.

The average reorientation time $\langle\tau\rangle$ decreases slowly as the temperature is increased. For low temperatures, the probe reorientation is accounted for by an Arrhenius law with an activation energy of 4-6 kJ/mol in the two PDMS samples (see Fig. 5). Close activation energy values were reported for PDMS investigated by neutron scattering and attributed to $\mathrm{CH}_{3}$ jumps about the $C_{3}$ axis $[82,83]$. This suggests a good coupling between the probe and local motions rather than the structural relaxation around $T_{g}$. The absence of any signature affecting $\langle\tau\rangle$ at $T_{g}$ suggests that around $T_{g}$ RAF is larger than MAF. No signature of cold crystallization is also found, thus indicating that the HF-EPR signal of the spin probe does not detect the formation of the crystals occurring on heating during data collection. In PDMSq, the trapped fraction of the spin probes is present between the cold crystallization and $T_{m}$, as indicated by the Arrhenius temperature dependence of the reorientation time of the trapped fraction $\tau_{\text {trapped }}$, observed up to $T_{m}$, which is in ideal continuation of the one of the single reorientation time $\tau_{\text {SRT }}$ characteristic of the spin probe reorientation at lower temperatures (see Fig. 5b). This is striking evidence that the probe molecules reorienting with the correlation time $\tau_{\text {trapped }}$ are located in RAF.

The average reorientation time $\langle\tau\rangle$ drops dramatically at about $213 \mathrm{~K}$, i.e., 17 $\mathrm{K}$ below $T_{m}$, for PDMSsc and at $T_{m}$ for PDMSq. At these temperatures, the distribution of reorientation times disappears, signaling the softening of RAF, and

(a)

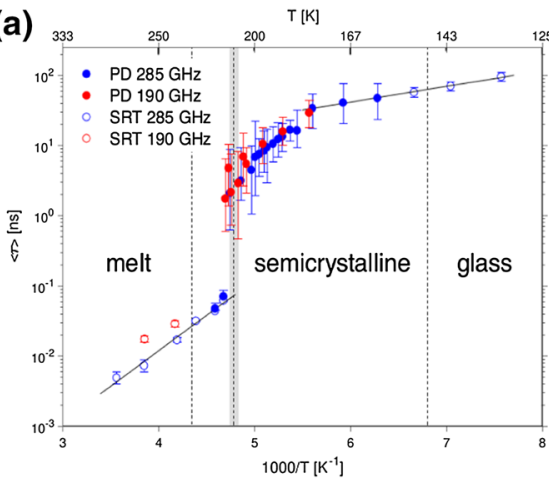

(b)

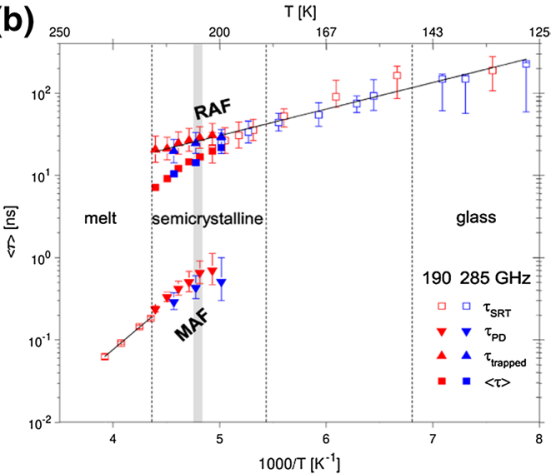

Fig. 5 Temperature dependence of the average reorientation time $\langle\tau\rangle$ of the spin probe in PDMSsc (lefthand side) [23] and in PDMSq (right-hand side) [24]. The dashed vertical lines mark the glass $(\simeq 147$ $\mathrm{K})$ and the melting transitions $(\simeq 230 \mathrm{~K})$. The gray region highlights the range of the onset of the PDMS melting $(\simeq 209 \mathrm{~K})$ as detected by DSC. The low-temperature and the high-temperature straight lines are Arrhenius fits with activation energies $4.4 \pm 0.3$ and $18.8 \pm 0.9 \mathrm{~kJ} / \mathrm{mol}$ for PDMSsc and $6.2 \pm 0.3$ and $20.9 \pm 0.4 \mathrm{~kJ} / \mathrm{mol}$ for PDMSq 
the SRT model applies. The precocious softening of RAF in PDMSsc might be ascribed to the melting of the thinnest lamellae releasing part of the constraints.

In the melt, the temperature dependence of $\langle\tau\rangle$ of the spin probe is described by an Arrhenius law with activation energy about $19 \mathrm{~kJ} / \mathrm{mol}$ in both samples. The activation energy is comparable to the one of the PDMS segmental dynamics (14.6 kJ/mol [82]), i.e., the spin probe is more coupled to the structural relaxation above the melting temperature than around $T_{g}$.

One should notice that the shortest reorientation time of the power distribution, $\tau_{\mathrm{PD}}$, approaches smoothly the single reorientation time found at temperatures above that where $\langle\tau\rangle$ drops. This is shown for PDMSq in Fig. 5b. This is evidence that the fastest fraction of the spin probes couples to the PDMS segmental motion, revealing the presence of MAF. This fast fraction accelerates without sensing the melting of the polymer.

\subsection{Local Reversible Melting}

The larger amount of RAF in PDMSq revealed signatures of reversible local melting [24]. The trapped and the more mobile fractions of the spin probe in the noncrystalline region of the polymer above $T_{g}$, described in the paragraph 4.2, are subject to a dynamic exchange process. We tentatively model it as a chemical reaction thermodynamically equilibrated, and consider the trapped and the more mobile fractions of the spin probe as the reactant and product, respectively. This scenario is sketched in the inset of Fig. 6. The related reaction equilibrium constant is $K_{e q}$.

Figure 6 presents the van't Hoff plot of the equilibrium constant $K_{\text {eq }}$. It is seen that the detrapping of the spin probe is favored; i.e., $K_{\text {eq }}$ is larger than 1 , if $T \gtrsim 209 \mathrm{~K}$, namely at temperatures higher than the onset of PDMS melting as detected by DSC which occurs at $\simeq 209 \mathrm{~K}$. Reminding that $K_{\text {eq }}=e^{\left(-\Delta G_{r}^{0} / R T\right)}$, one finds that the best-fit values of the standard Gibbs enthalpy and entropy of reaction drawn from Fig. 6 are $\Delta H_{r}^{0}=18 \pm 1 \mathrm{~kJ}$ and $\Delta S_{r}^{0}=86 \pm 5 \mathrm{~J} / \mathrm{K}$ per mole of spin probe. Assuming that the spin probes are very diluted, it should be observed that these parameters are dominated by the environments of the probe close to and far from the crystalline region and much less affected by the coupling of the spin probe with them. Therefore, picturing the environments as crystalline-like and liquid-like, we explore the proportionality between the van't Hoff parameters $\Delta H_{r}^{0}$ and $\Delta S_{r}^{0}$ with the enthalpy and entropy of fusion per repeating PDMS unit, $\Delta H_{m}$ and $\Delta S_{m}$, respectively:

$$
\begin{gathered}
\Delta H_{r}^{0}=z_{H} \Delta H_{m} \\
\Delta S_{r}^{0}=z_{S} \Delta S_{m},
\end{gathered}
$$

where $z_{H}$ and $z_{S}$ are suitable constants depending on the microscopic features of the exchange process. We take $\Delta H_{m}=4.619 \mathrm{~kJ} / \mathrm{mol}$ and $\Delta S_{m}=19.6 \mathrm{~J} /(\mathrm{K} \mathrm{mol})$ as listed in [84]. These values are in good agreement with a recent NMR study $\left(\Delta H_{m}=4.54\right.$ $\mathrm{kJ} / \mathrm{mol}[85])$ and alternative sources $\left(\Delta S_{m}=19.1 \mathrm{~J} /(\mathrm{K} \mathrm{mol})\right.$ [86] $)$. One finds $z_{H}=3.9$ and $z_{S}=4.4$ from Eq. (4) and Eq. (5), respectively; i.e., the structural 


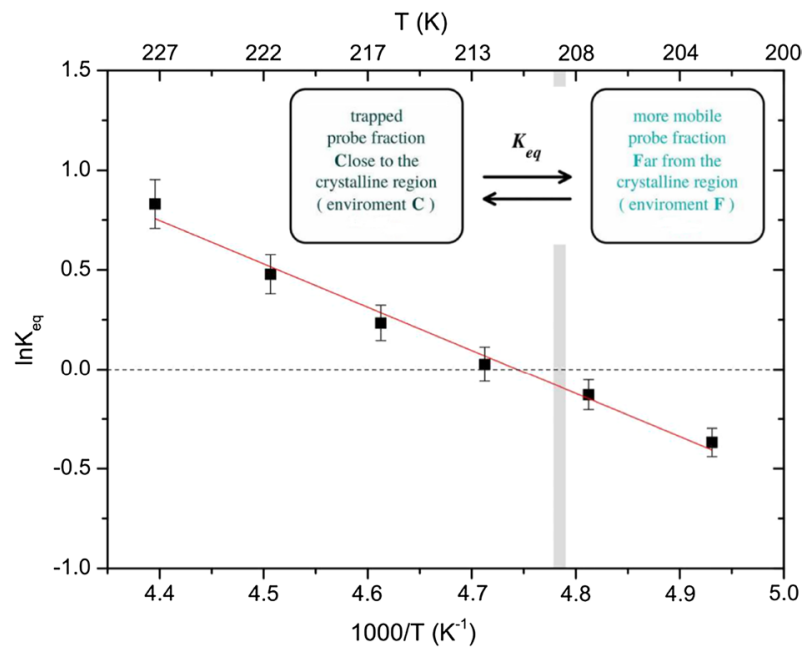

Fig. 6 Van't Hoff plot of the equilibrium constant $K_{\text {eq }}$ between the trapped and the more mobile fractions of the spin probe in semicrystalline PDMS, see ref. [24] for details. The straight red line is the best fit with the equation $K_{\mathrm{eq}}=e^{-\left(\Delta G_{r}^{0} / R T\right)}$ and $\Delta G_{r}^{0}=\Delta H_{r}^{0}-T \Delta S_{r}^{0}$. Detrapping involves positive standard enthalpy $\left(\Delta H_{r}^{0}=18 \pm 1 \mathrm{~kJ} / \mathrm{mol}\right)$ and entropy $\left(\Delta S_{r}^{0}=86 \pm 5 \mathrm{~J} /(\mathrm{K} \mathrm{mol})\right.$ of reaction. The gray region highlights the range of the onset of PDMS melting according to DSC $(\simeq 209 \mathrm{~K})$. Notice that detrapping is favored, i.e., $\ln K_{e q}$ is positive and $\Delta G_{r}^{0}$ is negative, if $T \gtrsim 209 \mathrm{~K}$. Inset: equilibrium between the fractions of the spin probes trapped and more mobile, being close to and far from the crystalline region, respectively

change of the surroundings of the spin probe from the trapped to the more mobile state is equivalent to the one of reversible melting of about $\mathrm{z} \simeq 4$ PDMS monomers.

\section{Conclusions}

We have reviewed studies carried out by HF-EPR spectroscopy concerning amorphous polymers, glassforming viscous liquids, polymer melts, and semicrystalline polymers. They evidence that HF-EPR spectroscopy provides novel insight into two intensely investigated hallmarks of disordered systems, namely the topography of the energy landscape and the dynamical heterogeneity. The energy landscape is probed by sensing: (1) the energy barriers which must be overcome by the spin probe during the reorientation process in glassy polymers and (2) the sharp changes in the reorientation of the spin probe in the region of the critical temperature $T_{c}$ predicted by the ideal mode-coupling theory (IMCT). Instead, dynamical heterogeneity is revealed by the wide distribution of reorientation times of the spin probes being dissolved in semicrystalline polymers and located in the disordered fraction far from the crystallites and trapped close to the crystallites. 
Funding Open access funding provided by Universit ̃̃ di Pisa within the CRUI-CARE Agreement.

Open Access This article is licensed under a Creative Commons Attribution 4.0 International License, which permits use, sharing, adaptation, distribution and reproduction in any medium or format, as long as you give appropriate credit to the original author(s) and the source, provide a link to the Creative Commons licence, and indicate if changes were made. The images or other third party material in this article are included in the article's Creative Commons licence, unless indicated otherwise in a credit line to the material. If material is not included in the article's Creative Commons licence and your intended use is not permitted by statutory regulation or exceeds the permitted use, you will need to obtain permission directly from the copyright holder. To view a copy of this licence, visit http://creativecommons.org/licen ses/by/4.0/.

\section{References}

1. L.J. Berliner, Spin Labeling: Theory and Applications (Academic, New York, 1976)

2. L.J. Berliner, J. Reuben, Biological Magnetic Resonance, vol. 8 (Plenum, New York, 1989)

3. K. Möbius, A. Savitsky, High-Field EPR Spectroscopy on Proteins and their Model Systems (The Royal Society of Chemistry, London, 2008)

4. P.P. Borbat, A.J. Costa-Filho, K.A. Earle, J.K. Moscicki, J.H. Freed, Science 291(5502), 266 (2001)

5. A. Savitsky, K. Möbius, Photosynth. Res. 102(2), 311 (2009)

6. D. Marsh, D. Kurad, V.A. Livshits, Magn. Reson. Chem. 43(S1), S20 (2005)

7. K.U.R. Naveed, L. Wang, H. Yu, R.S. Ullah, M. Haroon, S. Fahad, J. Li, T. Elshaarani, R.U. Khan, A. Nazir, Polym. Chem. 9, 3306 (2018)

8. C. Bährle, T.U. Nick, M. Bennati, G. Jeschke, F. Vogel, J. Phys. Chem. A 119(24), 6475 (2015)

9. V. Krinichnyi, Multi Frequency EPR Spectroscopy of Conjugated Polymers and their Nanocomposites (CRC Press, Boca Raton, 2017)

10. S.B. Orlinskii, H. Blok, E.J.J. Groenen, J. Schmidt, P.G. Baranov, C.M. de Donegá, A. Meijerink, Magn. Reson. Chem. 43(S1), S140 (2005)

11. C. Knapp, N. Weiden, K.P. Dinse, Magn. Reson. Chem. 43(S1), S199 (2005)

12. J. Telser, J. van Slageren, S. Vongtragool, M. Dressel, W.M. Reiff, S.A. Zvyagin, A. Ozarowski, J. Krzystek, Magn. Reson. Chem. 43(S1), S130 (2005)

13. J.H. Freed, Annu. Rev. Phys. Chem. 51, 655 (2000)

14. V. Bercu, M. Martinelli, C.A. Massa, L. Pardi, D. Leporini, J. Phys. Condens. Matter 16, L479 (2004)

15. V. Bercu, M. Martinelli, C.A. Massa, L.A. Pardi, D. Leporini, J. Chem. Phys. 123, 174906 (2005)

16. V. Bercu, M. Martinelli, C.A. Massa, L.A. Pardi, D. Leporini, Europhys. Lett. 72, 590 (2005)

17. V. Bercu, M. Martinelli, C.A. Massa, L.A. Pardi, D. Leporini, J. Non-Cryst, Solids 352, 5029 (2006)

18. V. Bercu, M. Martinelli, C.A. Massa, L.A. Pardi, D. Leporini, Philos. Mag. 87, 795 (2007)

19. V. Bercu, M. Martinelli, C.A. Massa, L.A. Pardi, D. Leporini, Appl. Magn. Reson. 33, 365 (2008)

20. V. Bercu, M. Martinelli, C.A. Massa, L.A. Pardi, D. Leporini, Z. Phys. Chem. 226, 1379 (2012)

21. V. Bercu, M. Martinelli, C.A. Massa, L.A. Pardi, D. Leporini, J. Optoelectron. Adv. Mater. 9, 1785 (2007)

22. V. Bercu, M. Martinelli, C.A. Massa, L.A. Pardi, E. Rössler, D. Leporini, J. Chem. Phys. 129, 081102 (2008)

23. C.A. Massa, S. Pizzanelli, V. Bercu, L. Pardi, D. Leporini, Macromolecules 47, 6748 (2014)

24. C.A. Massa, S. Pizzanelli, V. Bercu, L. Pardi, D. Leporini, Macromolecules 50, 5061 (2017)

25. M. Martinelli, C.A. Massa, L. Pardi, V. Bercu, F.F. Popescu, Phys. Rev. B 67, 014425 (2004)

26. A. Caneschi, A. Dei, D. Gatteschi, C.A. Massa, L.A. Pardi, S. Poussereau, L. Sorace, Chem. Phys. Lett. 371, 694 (2003)

27. F.F. Popescu, M. Martinelli, C.A. Massa, L.A. Pardi, V. Bercu, Magn. Reson. Chem. 43, S215 (2005) 
28. F.F. Popescu, V. Bercu, J.N. Barascu, M. Martinelli, C.A. Massa, L.A. Pardi, M. Stefan, S.V. Nistor, M. Nikl, P. Bohacek, J. Chem. Phys. 128, 034505 (2008)

29. F.F. Popescu, V. Bercu, J.N. Barascu, M. Martinelli, C.A. Massa, L.A. Pardi, M. Stefan, S.V. Nistor, M. Nikl, Opt. Mater. 32, 570 (2010)

30. F. DiBenedetto, D. Borrini, A. Caneschi, G. Fornaciai, M. Innocenti, A. Lavacchi, C.A. Massa, G. Montegrossi, L.A. Pardi, M. Romanelli, Phys. Chem. Miner. 38, 483 (2011)

31. F. DiBenedetto, A. Buccianti, G. Montegrossi, M. Innocenti, C.A. Massa, L.A. Pardi, M. Romanelli, Am. Miner. 97, 1619 (2012)

32. L.C. Brunel, A. Caneschi, A. Dei, D. Friselli, D. Gatteschi, A.K. Hassan, L. Lenci, M. Martinelli, C.A. Massa, L.A. Pardi, F.F. Popescu, I. Ricci, L. Sorace, Res. Chem. Intermed. 28, 215 (2002)

33. G. Annino, M. Cassettari, M. Fittipaldi, L. Lenci, I. Longo, M. Martinelli, C.A. Massa, L.A. Pardi, Appl. Magn. Reson. 506, 495 (2000)

34. D. Friselli, C.A. Massa, M. Martinelli, L. Pardi, I. Ricci, Inorg. Chim. Acta 361, 4164 (2008)

35. L. Lenci, M. Martinelli, C.A. Massa, L.A. Pardi, I. Ricci, A.K. Hassan, A. Caneschi, L.C. Brunel, Appl. Magn. Reson. 21, 607 (2001)

36. M.D. Ediger, Annu. Rev. Phys. Chem. 51, 99 (2000)

37. R. Richert, J. Phys. Condens. Matter 14, R703 (2002)

38. D. Leporini, V. Schädler, U. Wiesner, H.W. Spiess, G. Jeschke, J. Chem. Phys. 119, 11829 (2003)

39. M. Faetti, M. Giordano, D. Leporini, L. Pardi, Macromolecules 32, 1876 (1999)

40. C. Angell, J. Non-Cryst. Solids 131-133, 13 (1991)

41. P.G. Debenedetti, Metastable Liquids: Concepts and Principles (Princeton University Press, Princeton, 1996), p. 411

42. K.L. Ngai, Relaxation and Diffusion in Complex Systems (Springer, Berlin, 2011)

43. A. Ottochian, D. Leporini, Philos. Mag. 91, 1786 (2011)

44. I. Chang, F. Fujara, B. Geil, G. Heuberger, T. Mangel, H. Sillescu, J. Non Cryst. Solids 172-175, 248 (1994)

45. J. Douglas, D. Leporini, J. Non-Cryst. Solids 235-237, 137 (1998)

46. H. Sillescu, J. Non-Cryst. Solids 243(2), 81 (1999)

47. L. Berthier, G. Biroli, Rev. Mod. Phys. 83, 587 (2011)

48. F.H. Stillinger, Energy Landscapes, Inherent Structures, and Condensed-Matter Phenomena (Princeton University Press, Princeton, 2015)

49. C. Monthus, J.P. Bouchaud, J. Phys. A Math. Gen. 29, 3847 (1996)

50. G.R. Strobl, The Physics of Polymers, 3rd edn. (Springer, Berlin, 2007)

51. B. Wunderlich, Prog. Polym. Sci. 28, 383 (2003)

52. G. Strobl, Prog. Polym. Sci. 31, 398 (2006)

53. K.U. Kirst, F. Kremer, V.M. Litvinov, Macromolecules 26, 975 (1993)

54. K. Schmidt-Rohr, H.W. Spiess, Multidimensional Solid-State NMR and Polymers (Academic Press, London, 1994)

55. R.F. Boyer, Polymer 17, 996 (1976)

56. P. Törmälä, J. Macromol. Sci. Polym. Rev. 17, 297 (1979)

57. N.G. McCrum, B.E. Read, G. Williams, Anelastic and Dielectric Effects in Polymeric Solids (Dover Publications, New York, 1991)

58. K.L. Ngai, Relaxation and Diffusion in Complex Systems (Springer, Berlin, 2011)

59. K.L. Ngai, C.M. Roland, Macromolecules 26, 2688 (1993)

60. L. Larini, A. Barbieri, D. Prevosto, P.A. Rolla, D. Leporini, J. Phys. Condens. Matter 17, L199 (2005)

61. A. Wurm, M. Merzlyakov, C. Schick, J. Therm. Anal. Calorim. 60, 807 (2000)

62. B. Wunderlich, Thermal Analysis of Polymeric Materials (Springer, Berlin, 2005)

63. K.A. Topp, D.G. Cahill, Z. Phys. B 101, 235 (1996)

64. A.P. Sokolov, V.N. Novikov, B. Strube, Europhys. Lett. 38, 49 (1997)

65. N.V. Surovtsev, J.A.H. Wiedersich, V.N. Novikov, E. Rössler, A.P. Sokolov, Phys. Rev. B 58, 14888 (1998)

66. A. Barbieri, G. Gorini, D. Leporini, Phys. Rev. E 69, 061509 (2004)

67. L. Andreozzi, F. Cianflone, C. Donati, D. Leporini, J. Phys. Condens. Matter 8, 3795 (1996)

68. J. Kim, T. Keyes, J. Chem. Phys. 121, 4237 (2004)

69. C. De Michele, D. Leporini, Phys. Rev. E 63, 036702 (2001) 
70. W. Götze, Complex Dynamics of Glass-Forming Liquids: A Mode-Coupling Theory (Oxford University Press, Oxford, 2008)

71. C.A. Angell, in Complex Behavior in Glassy Systems, ed. by M. Rubi, C. Perez-Vicente (Springer, Berlin, 1997) pp. 1-21

72. S. Sastry, P.G. Debenedetti, F.H. Stillinger, Nature 393, 554 (1998)

73. K.A. Earle, J.K. Moscicki, A. Polimeno, J.H. Freed, J. Chem. Phys. 106, 9996 (1997)

74. M. Giordano, P. Grigolini, D. Leporini, P. Marin, Phys. Rev. A 28, 2474 (1983)

75. A. Tölle, H. Schober, J. Wuttke, F. Fujara, Phys. Rev. E 56, 809 (1997)

76. H. Meyer, in Polymer Crystallization: Observations, Concepts and Interpretations, vol. 10, ed. by G. Reiter, J.U. Sommer (Springer, Berlin, 2008) pp. 177-195

77. X. Wang, J. Ouyang, Int. J. Chem. Eng. Appl. 6, 2831 (2015)

78. H. St-Onge, IEEE Trans. Electr. Insul. EI-15, 359 (1980)

79. J. Mark, K. Ngai, W. Graessley, L. Mandelkern, E. Samulski, J. Koenig, G. Wignall, in Physical Properties of Polymers, 3rd edn. (Cambridge University Press, Cambridge, 2004), pp. 209-315. https://doi.org/10.1017/CBO9781139165167.005

80. E. Ivanov, N. Sov. Phys. JETP 18, 1041 (1964)

81. L. Alessi, L. Andreozzi, M. Faetti, D. Leporini, J. Chem. Phys. 114, 3631-3639 (2001)

82. V. Arrighi, S. Gagliardi, C. Zhang, F. Ganazzoli, J.S. Higgins, R. Ocone, M.T.F. Telling, Macromolecules 36, 8738 (2003)

83. H.H. Grapengeter, B. Alefeld, R. Kosfeld, Colloid Polym. Sci. 265, 226 (1987)

84. L. Mandelkern, R.G. Alamo, in Physical Properties of Polymers Handbook, 2 edn, ed. by J.E. Mark (Springer, Berlin, 2007), pp. 165-186

85. A. Maus, K. Saalwächter, Macromol. Chem. Phys. 208, 2066 (2007)

86. V.P. Privalko, J. Phys. Chem. 84, 3307 (1980)

Publisher's Note Springer Nature remains neutral with regard to jurisdictional claims in published maps and institutional affiliations. 